\title{
Spin-dependent localized Hartree-Fock density-functional calculation of singly, doubly, and triply excited and Rydberg states of He- and Li-like ions
}

\author{
Zhongyuan Zhou ${ }^{1,2}$ and Shih-I Chu ${ }^{1}$ \\ ${ }^{1}$ Department of Chemistry, University of Kansas, Lawrence, Kansas 66045, USA \\ ${ }^{2}$ Department of Physics and Astronomy, University of Kansas, Lawrence, Kansas 66045, USA
}

(Received 7 July 2004; published 28 February 2005)

\begin{abstract}
A spin-dependent density-functional approach for the calculation of highly and multiply excited state of atomic system is proposed based on the localized Hartree-Fock density-functional method and Slater's diagonal sum rule. In this approach, electron spin orbitals in an electronic configuration are obtained first by solving the Kohn-Sham equation with an exact nonvariational spin-dependent localized Hartree-Fock exchange potential. Then a single-Slater-determinant energy of the electronic configuration is calculated by using these electron spin orbitals. Finally, a multiplet energy of an excited state is evaluated from the single-Slater-determinant energies of the electronic configurations involved in terms of Slater's diagonal sum rule. This approach has been applied to the calculation of singly, doubly, and especially triply excited Rydberg states of He- and Li-like ions. The total energies obtained from the calculation with an exchange-only ( $X$-only) potential are surprisingly close to those of Hartree-Fock method and the total energies from the calculation with exchange-correlation potential are in overall agreement with available theoretical and experimental data. The presented procedure provides a simple and computationally efficient scheme for the accurate calculation of highly and multiply excited Rydberg states of an atomic system within density-functional theory.
\end{abstract}

DOI: 10.1103/PhysRevA.71.022513

PACS number(s): 31.15.Ew, 32.80.Wr, 32.80.Rm

\section{INTRODUCTION}

Density-functional theory (DFT) $[1,2]$ has been widely applied to many areas in theoretical physics and chemistry $[3,4]$ as a powerful $a b$ initio approach of calculating the ground-state properties of many-electron systems due to its computational simplicity in dealing with systems having a large number of electrons. The basis of DFT is the Hohenberg and Kohn (HK) theorem [1] and the Kohn-Sham (KS) equation [2]. The most important and troublesome part in DFT is the exchange-correlation (XC) potential because it determines not only calculation accuracy but also the usefulness of a DFT calculation [5].

In a traditional approximation, such as the local density approximation (LDA) $[3,4]$ and the generalized gradient approximation (GGA) [6-8], the XC potential is obtained by using uniform electron gas. Because of the incomplete cancellation of spurious self-interactions [9], the approximate $\mathrm{XC}$ potential falls off too fast and thus exhibits some incorrect asymptotic behaviors [10]. As a result, no Rydberg series and only a few bound unoccupied states can be available from a calculation with this kind of XC potential. In addition, the traditional XC potential has an inherent degeneracy due to its independence of symmetries such as orbital angular momentum and spin of the state considered and thus the calculated energies for different symmetries may have the same value. Therefore, the KS equation with traditional XC potential cannot be directly applied to the calculation of excited-state energy, especially highly excited-state energy.

In fact, DFT itself is a ground-state theory. KS eigenvalue differences of unoccupied and occupied orbitals are not rigorously defined as excitation energies. One needs to go beyond the standard DFT, for example, using a time-dependent DFT approach [11-13] to calculate excitation energy. How- ever, KS eigenvalues can serve as good zero-order excitedstate energies provided that they are gained by solving the KS equation with a high-quality XC potential [14]. A number of theoretical methods have been developed by adopting this point of view (see a recent review article [15] for more approaches), in which the work-function- (WF-) based exchange potential approach [16-19], the open-shell localized Hartree-Fock (LHF) density-functional approach [20,21], and the multireference LHF density-functional approach $[22,23]$ have drawn more attention because of their successful applications to the calculations of atomic and molecular excited states.

However, almost all the calculations using the timedependent DFT approach, open-shell LHF approach, and multireference LHF approach so far are performed for lower singly excited states. Although better results from calculations of the WF method were reported for both singly and doubly excited states of atomic systems $[18,19]$, one needs the rotational component of the field in computation, which, in general, is very hard to calculate. In the atomic case, it may be neglected in comparison with the irrotational component, but in the molecular case, it cannot be ignored due to the departure of electronic structure from spherical symmetry [15]. Even for the atomic case, WF calculation often encounters a self-consistent convergence problem for high-lying Rydberg excited states [19]. Therefore, an effective, simple, and easy-to-be-applied density-functional method for the calculation of highly and multiply excited states remains to be explored.

In this paper, we present a spin-dependent densityfunctional approach for the calculation of highly and multiply excited states of atomic systems based on the LHF density-functional method $[14,24]$ and Slater's diagonal sum rule [25]. In this approach, the exchange potential used is an 
exact nonvariational spin-dependent localized Hartree-Fock (SLHF) exchange potential constructed by extending the ground-state LHF exchange potential to excited states, electron spin orbitals in an electronic configuration are obtained by solving KS equation with the SLHF exchange potential, a single-Slater-determinant energy of the electronic configuration is calculated by using these electron spin orbitals, and a multiplet energy of an excited state is evaluated from the single-Slater-determinant energies of the electronic configurations involved in terms of Slater's diagonal sum rule. The SLHF exchange potential is free of Coulomb selfinteractions, dependent on symmetries of the state under consideration, requires occupied orbitals only, and exhibits correct long-range behaviors; it can be used to calculate the spin orbitals and density for each individual excited state and thus is promising for the calculation of highly and multiply excited states of atomic system. We have applied this procedure to the calculations of singly, doubly, and especially triply excited states of He- and Li-like ions. Due to the singularity at the origin and long-range nature of the Coulomb potential, we use the generalized pseudospectral (GPS) method [26] to discretize spatial coordinates and optimize solution of the KS equation so as to obtain accurate eigenvalues and density functionals. The total energies obtained from the calculation with an exchange-only (X-only) potential (X-only calculation) are found to be surprisingly close to those of the Hartree-Fock (HF) method and the total energies from the calculation with the $\mathrm{XC}$ potential (XC calculation) are in overall agreement with available theoretical and experimental data.

\section{THEORETICAL METHOD}

\section{A. Spin-dependent localized Hartree-Fock density-functional method}

In spin-dependent DFT, the spin-dependent electron density $\rho_{\sigma}(\mathbf{r})$ is defined by

$$
\rho_{\sigma}(\mathbf{r})=\sum_{i=1}^{N_{\sigma}} w_{i \sigma}\left|\varphi_{i \sigma}(\mathbf{r})\right|^{2},
$$

where $\sigma$ is the electron spin, equal to $\alpha$ and $\beta$ for spin-up and spin-down, $N_{\sigma}$ is the number of electrons with spin $\sigma$, $\varphi_{i \sigma}(\mathbf{r})$ is the $i$ th spin orbital with spin $\sigma$, and $w_{i \sigma}$ is the occupied number of electrons in the spin orbital $\varphi_{i \sigma}(\mathbf{r})$. The electron spin orbital $\varphi_{i \sigma}(\mathbf{r})$ is determined by the KS equation

$$
H_{\sigma}(\mathbf{r}) \varphi_{i \sigma}(\mathbf{r})=\varepsilon_{i \sigma} \varphi_{i \sigma}(\mathbf{r}),
$$

with the KS Hamiltonian $H_{\sigma}(\mathbf{r})$ given by

$$
H_{\sigma}(\mathbf{r})=-\frac{1}{2} \nabla^{2}+V_{\sigma}^{\mathrm{eff}}(\mathbf{r}),
$$

and the local effective potential $V_{\sigma}^{\mathrm{eff}}(\mathbf{r})$ given by

$$
V_{\sigma}^{\mathrm{eff}}(\mathbf{r})=V_{e x t}(\mathbf{r})+V_{H}(\mathbf{r})+V_{x c \sigma}(\mathbf{r}),
$$

where $V_{\text {ext }}(\mathbf{r})$ is the external potential, $V_{H}(\mathbf{r})$ is the Hartree potential (classical Coulomb electrostatic potential between electrons), and $V_{x c \sigma}(\mathbf{r})$ is the XC potential. The external potential is known accurately for a given atomic system. The
Hartree potential can be calculated exactly by

$$
V_{H}(\mathbf{r})=\int \frac{\rho\left(\mathbf{r}^{\prime}\right)}{\left|\mathbf{r}-\mathbf{r}^{\prime}\right|} d \mathbf{r}^{\prime},
$$

where $\rho(\mathbf{r})=\rho_{\alpha}(\mathbf{r})+\rho_{\beta}(\mathbf{r})$ is the total electron density. Thus finding a suitable $\mathrm{XC}$ potential is the key to the calculation of both the ground state and excited states. The XC potential can be divided into the exchange potential $V_{x \sigma}(\mathbf{r})$ and the correlation potential $V_{c \sigma}(\mathbf{r})$.

Recently, the X-only LHF density-functional method has been proposed and successfully applied to the ground-state calculation of atomic and molecular systems [14,24]. In that method, electron orbitals are calculated by solving the KS equation with the LHF exchange potential as its exchange potential. The LHF exchange potential is derived under the assumption that the $\mathrm{X}$-only $\mathrm{KS}$ determinant is equal to the HF determinant. This potential is free of Coulomb selfinteractions, requires only occupied orbitals, and exhibits correct long-range behaviors. The exchange potential in the Krieger, Li, and Iafrate (KLI) procedure [27,28], an approximation of the exact exchange (EEX) optimized effective potential (OEP) [29], is an approximation to the LHF exchange potential.

In this paper, we extend the LHF exchange potential to the excited-state calculation of the atomic system by assuming that the electron spin orbitals of excited states are determined by the KS equation of a noninteracting system and the $\mathrm{X}$-only KS determinant of the noninteracting system equals the HF determinant of the interacting system for the excited states. Based on this postulate, following a similar procedure used in Ref. [14], we obtain the spin-dependent localized Hartree-Fock (SLHF) exchange potential

$$
V_{x \sigma}^{\mathrm{SLHF}}(\mathbf{r})=V_{x \sigma}^{\mathrm{S}}(\mathbf{r})+V_{x \sigma}^{\mathrm{C}}(\mathbf{r}),
$$

where the first term $V_{x \sigma}^{\mathrm{S}}(\mathbf{r})$ is the Slater potential [25] and the second term $V_{x \sigma}^{\mathrm{C}}(\mathbf{r})$ is a correction to the Slater potential. Both of them are determined by occupied KS spin orbitals and are calculated by

$$
V_{x \sigma}^{\mathrm{S}}(\mathbf{r})=-\frac{1}{\rho_{\sigma}(\mathbf{r})} \sum_{i, j=1}^{N_{\sigma}} \gamma_{i j}^{\sigma}(\mathbf{r}) \int \frac{\gamma_{i j}^{\sigma}\left(\mathbf{r}^{\prime}\right)}{\left|\mathbf{r}-\mathbf{r}^{\prime}\right|} d \mathbf{r}^{\prime},
$$

and

$$
V_{x \sigma}^{\mathrm{C}}(\mathbf{r})=\frac{1}{\rho_{\sigma}(\mathbf{r})} \sum_{i, j=1}^{N_{\sigma}} \gamma_{i j}^{\sigma}(\mathbf{r}) Q_{i j}^{\sigma},
$$

where $\gamma_{i j}^{\sigma}(\mathbf{r})$ and $Q_{i j}^{\sigma}$ are defined by

$$
\gamma_{i j}^{\sigma}(\mathbf{r})=\varphi_{i \sigma}(\mathbf{r}) \varphi_{j \sigma}(\mathbf{r})
$$

and

$$
Q_{i j}^{\sigma}=\left\langle\varphi_{j \sigma}\left|V_{x \sigma}^{\mathrm{SLHF}}-V_{x \sigma}^{\mathrm{NL}}\right| \varphi_{i \sigma}\right\rangle .
$$

Here, $V_{x \sigma}^{\mathrm{NL}}$ is a nonlocal exchange operator of the form of the $\mathrm{HF}$ exchange potential but constructed from KS spin orbitals, $\left\langle\varphi_{j \sigma}\left|V_{x \sigma}^{\mathrm{SLHF}}\right| \varphi_{i \sigma}\right\rangle$ and $\left\langle\varphi_{j \sigma}\left|V_{x \sigma}^{\mathrm{NL}}\right| \varphi_{i \sigma}\right\rangle$ are symmetric matrix elements calculated by 


$$
\left\langle\varphi_{j \sigma}\left|V_{x \sigma}^{\mathrm{SLHF}}\right| \varphi_{i \sigma}\right\rangle=\int \gamma_{i j}^{\sigma}\left(\mathbf{r}^{\prime}\right) V_{x \sigma}^{\mathrm{SLHF}}\left(\mathbf{r}^{\prime}\right) d \mathbf{r}^{\prime}
$$

and

$$
\left\langle\varphi_{j \sigma}\left|V_{x \sigma}^{\mathrm{NL}}\right| \varphi_{i \sigma}\right\rangle=-\sum_{k=1}^{N_{\sigma}} \iint \frac{\gamma_{i k}^{\sigma}(\mathbf{r}) \gamma_{k j}^{\sigma}\left(\mathbf{r}^{\prime}\right)}{\left|\mathbf{r}-\mathbf{r}^{\prime}\right|} d \mathbf{r} d \mathbf{r}^{\prime} .
$$

In the spin-dependent DFT, the exchange interaction only occurs among electrons with the same spin. In Eq. (6), when $V_{x \sigma}^{\mathrm{C}}(\mathbf{r})=0$, the SLHF exchange potential is reduced to the Slater potential of the HF method [25]. Because $V_{x \sigma}^{\mathrm{C}}(\mathbf{r})$ depends on $V_{x \sigma}^{\mathrm{SLHF}}$, the SLHF exchange potential $V_{x \sigma}^{\mathrm{SLHF}}(\mathbf{r})$ has to be computed in a self-consistent manner. In Eq. (8), if the terms with $i \neq j$ are neglected, the SLHF exchange potential reduces to the KLI exchange potential [27,28].

For the spin-dependent DFT, SLHF exchange potentials determined by Eqs. (6)-(12) have two arbitrary additive constants. The physical orbitals can only be obtained by the use of appropriate constants in the exchange potential. To settle down the constants so as to pick up the physical orbitals, we demand the highest occupied orbital $N_{\sigma}$ of each spin $\sigma$ to satisfy [14]

$$
\left\langle\varphi_{N_{\sigma} \sigma}\left|V_{x \sigma}^{\mathrm{SLHF}}-V_{x \sigma}^{\mathrm{NL}}\right| \varphi_{N_{\sigma} \sigma}\right\rangle=0 .
$$

This condition indicates that the highest occupied orbital does not contribute to the correction term $V_{x \sigma}^{\mathrm{C}}(\mathbf{r})$. In this case, the correction term $V_{x \sigma}^{C}(\mathbf{r})$ decays exponentially, the SLHF exchange potential behaves asymptotically as the Slater potential, and thus approaches to $-1 / r$ at long range [14]. Moreover, the SLHF exchange potential depends only on the occupied orbitals and thus contains detailed information of the electronic configuration considered.

It should be noted that the SLHF exchange potential above is an extension of the ground-state LHF exchange potential although they are very similar to each other [14]. Since the SLHF formalism is not derived from a variational principle for energy, the variational restriction on the ground state being the lowest state of a given space-spin symmetry is not applicable here. Thus the calculation of spin orbitals for excited states by solving the KS equation (2) with the SLHF exchange potential is not subject to a variational bound for energy. Furthermore, unlike the WF method, the SLHF method guarantees the existence of a local effective potential for both the ground state and excited states [14,20]. Because of the nonvariational nature of the SLHF exchange potential, the KS equation (2) is no longer a variational equation and thus the problem of variational collapse to the ground state is avoided in our approach. This is a considerable advantage since, for a variational calculation of excitedstate energy, to satisfy the wave function orthogonality (to prevent variational collapse) can be a task of formidable complexity [19].

Calculation of the total energy should include the correlation effect. This effect is taken into account via correlation energy in the DFT calculation. Although quite a few approaches have been developed for this purpose, the secondorder gradient correlation potential and energy functional proposed by Lee, Yang, and Parr (LYP) [7] have been shown to provide an excellent representation of actual correlation energy and will be incorporated into our calculation to estimate the correlation effect.

\section{B. Central-field approach of atomic system}

To demonstrate the feasibility of the approach developed above, we apply it to the excited-state calculation of atomic system. To specify the spin-dependent property, an electron spin orbital is signified by three quantum numbers $n, l$, and $\sigma$, where $n$ and $l$ are the principal quantum number and orbital angular momentum quantum number of the electron, respectively. In spherical coordinates, a spin orbital $\varphi_{i \sigma}(\mathbf{r})$ of the electron with quantum numbers $n, l$, and $\sigma$ can be expressed by

$$
\varphi_{i \sigma}(\mathbf{r})=\frac{R_{n l \sigma}(r)}{r} Y_{l m}(\theta, \phi),
$$

where $R_{n l \sigma}(r)$ is the radial spin orbital, $Y_{l m}(\theta, \phi)$ is the spherical harmonic, $m$ is the azimuthal quantum number, and $i$ is a set of quantum numbers apart from spin $\sigma$ of the spin orbital.

The electron density is evaluated by substituting Eq. (14) into Eq. (1). For the atomic system investigated in this work, the system is described by a spherically averaged electron density given by

$$
\rho_{\sigma}(r)=\frac{1}{4 \pi} \int \rho_{\sigma}(\mathbf{r}) d \Omega=\frac{1}{4 \pi} \sum_{n l}^{\nu_{\sigma}} w_{n l \sigma}\left[\frac{R_{n l \sigma}}{r}\right]^{2},
$$

where the symbol $\nu_{\sigma}$ stands for a set of quantum numbers for summation and the sum is performed over all the occupied spin-orbitals with spin $\sigma$. The electron density obtained in this way is accurate for spherically symmetric (close-shell) states, but approximate for nonspherically symmetric (openshell) states. When this kind of electron density is used to evaluate the energy of a nonspherically symmetric state, it may induce an error. However, the error is negligible compared to the order of the calculated multiplet splitting [15].

Substituting Eq. (14) into Eq. (2), multiplying the equation by $Y_{l m}^{*}(\theta, \phi)$, integrating the equation over angles, and averaging the equation over $m$, finally, we obtain the radial KS equation for the radial spin orbital $R_{n l \sigma}(r)$,

$$
\left[-\frac{1}{2} \frac{d^{2}}{d r^{2}}+\frac{l(l+1)}{2 r^{2}}+v_{\sigma}^{\mathrm{eff}}(r)\right] R_{n l \sigma}=\varepsilon_{n l \sigma} R_{n l \sigma},
$$

where $v_{\sigma}^{\text {eff }}(r)$ is the radial effective potential given by

$$
\begin{aligned}
v_{\sigma}^{\mathrm{eff}}(r) & =\frac{1}{2 l+1} \sum_{m=-l}^{l} \int Y_{l m}^{*}(\theta, \phi) V_{\sigma}^{\mathrm{eff}}(\mathbf{r}) Y_{l m}(\theta, \phi) d \Omega \\
& =v_{e x t}(r)+v_{H}(r)+v_{x \sigma}^{\mathrm{SLHF}}(r)+v_{c \sigma}^{\mathrm{LYP}}(r) .
\end{aligned}
$$

Here, $v_{e x t}(r), v_{H}(r), v_{x \sigma}^{\mathrm{SLHF}}(r)$, and $v_{c \sigma}^{\mathrm{LYP}}(r)$ are the radial external potential, radial Hartree potential, radial SLHF exchange potential, and radial LYP correlation potential [7], respectively.

In an atomic system, the external potential is the Coulomb potential between the electron and the nucleus. For the atom 
with a nuclear charge $Z$, the radial external potential is given by

$$
v_{e x t}(r)=-\frac{Z}{r} .
$$

To calculate the radial Hartree potential, by applying the multipole expansion of $1 /\left|\mathbf{r}-\mathbf{r}^{\prime}\right|$, the orthonormality of spherical harmonics, and the spherical-harmonic expansion of two spherical harmonics to Eq. (5), we obtain [30]

$$
v_{H}(r)=4 \pi \int \frac{1}{r_{>}} \rho\left(r^{\prime}\right) r^{\prime 2} d r^{\prime},
$$

where $r_{>}$is the larger of $r$ and $r^{\prime}$, and $\rho(r)=\rho_{\alpha}(r)+\rho_{\beta}(r)$ is the spherically averaged total electron density.

Similarly, from Eqs. (6)-(12), we obtain the radial SLHF exchange potential

$$
v_{x \sigma}^{\mathrm{SLHF}}(r)=v_{x \sigma}^{\mathrm{S}}(r)+v_{x \sigma}^{\mathrm{C}}(r),
$$

where the radial Slater potential is given by

$$
v_{x \sigma}^{\mathrm{S}}(r)=-\frac{1}{4 \pi \rho_{\sigma}(r)} \sum_{n l m}^{\nu_{\sigma}} \sum_{n^{\prime} l^{\prime} m^{\prime}}^{\nu_{\sigma}} s_{n l m, n^{\prime} l^{\prime} m^{\prime}}^{\sigma}(r),
$$

and the radial correction is given by

$$
v_{x \sigma}^{\mathrm{C}}(r)=\frac{1}{4 \pi \rho_{\sigma}(r)} \sum_{n l m}^{\nu_{\sigma}} \sum_{n^{\prime} l^{\prime} m^{\prime}}^{\nu_{\sigma}} c_{n l m, n^{\prime} l^{\prime} m^{\prime}}^{\sigma}(r) .
$$

In Eqs. (21) and (22),

$$
\begin{gathered}
s_{n l m, n^{\prime} l^{\prime} m^{\prime}}^{\sigma}(r)=p_{n l \sigma, n^{\prime} l^{\prime} \sigma}(r) \sum_{k} \lambda_{l m, l^{\prime} m^{\prime}}^{k} q_{n l \sigma, n^{\prime} l^{\prime} \sigma}^{k}(r), \\
c_{n l m, n^{\prime} l^{\prime} m^{\prime}}^{\sigma}(r)=p_{n l \sigma, n^{\prime} l^{\prime} \sigma}(r) \delta_{l l^{\prime}} \delta_{m m^{\prime}} \mu_{n l, n^{\prime} l^{\prime}}^{\sigma m^{\prime}}, \\
p_{n l \sigma, n^{\prime} l^{\prime} \sigma^{\prime}}(r)=\frac{R_{n l \sigma}(r) R_{n^{\prime} l^{\prime} \sigma^{\prime}}(r)}{r^{2}}, \\
q_{n l \sigma, n^{\prime} l^{\prime} \sigma^{\prime}}^{k}(r)=\int \frac{r_{<}^{k}}{r_{>}^{k+1}} p_{n l \sigma, n^{\prime} l^{\prime} \sigma^{\prime}}\left(r^{\prime}\right) r^{\prime 2} d r^{\prime}, \\
\lambda_{l m, l^{\prime} m^{\prime}}^{k}=(-1)^{m^{\prime}-m}\left\langle l m k m^{\prime}-m \mid l^{\prime} m^{\prime}\right\rangle\left\langle l^{\prime} m^{\prime} k m-m^{\prime} \mid l m\right\rangle \\
\quad \times\left\langle l 0 k 0 \mid l^{\prime} 0\right\rangle\left\langle l^{\prime} 0 k 0 \mid l 0\right\rangle,
\end{gathered}
$$

and

$$
\begin{aligned}
\mu_{n l, n^{\prime} l^{\prime}}^{\sigma m^{\prime}}= & \sum_{n_{0} l_{0} m_{0}}^{\nu_{\sigma}} \int p_{n l \sigma, n_{0} l_{0} \sigma^{(}}\left(r^{\prime}\right)\left[v_{x \sigma}^{\mathrm{SLHF}}\left(r^{\prime}\right) \delta_{n^{\prime} n_{0}} \delta_{l^{\prime} l_{0}}\right. \\
& \left.+\sum_{k} \lambda_{l_{0} m_{0}, l^{\prime} m^{\prime}}^{k} q_{n_{0} l_{0} \sigma, n^{\prime} l^{\prime} \sigma}^{k}\left(r^{\prime}\right)\right] r^{\prime 2} d r^{\prime} .
\end{aligned}
$$

In Eq. (27), the symbol $\langle\cdots \mid \cdots\rangle$ is the Clebsch-Gordan coefficient. According to the property of the Clebsch-Gordan coefficient, $k=\left|l-l^{\prime}\right|,\left|l-l^{\prime}\right|+1, \ldots, l+l^{\prime}$ and $l+l^{\prime}+k=$ even.

\section{Generalized pseudospectral method and optimal solution of the KS equation}

In order to solve the radial KS equation, we use the generalized pseudospectral (GPS) method [26] to discretize Eq. (16). The GPS method is a kind of collocation-cardinal function method. The basic idea of this method is to approximate an exact function $f(x)$ defined on the interval $[-1,1]$ by an $N$ th-order polynomial $f_{N}(x)$ constructed by a cardinal function $g_{j}(x)$,

$$
f(x) \simeq f_{N}(x) \equiv \sum_{j=0}^{N} f\left(x_{j}\right) g_{j}(x),
$$

and ensure the approximation to be exact at the collocation point $x_{i}$, namely $f_{N}\left(x_{i}\right) \equiv f\left(x_{i}\right)$. This requires that the cardinal function satisfies $g_{j}\left(x_{i}\right)=\delta_{i j}$.

In the Legendre generalized pseudospectral (LGPS) method used in this work, the cardinal function $g_{j}(x)$ is constructed by the $N$ th-order Legendre polynomial $P_{N}(x)$ and its first derivative $\dot{P}_{N}(x)$

$$
g_{j}(x)=-\frac{1}{N(N+1) P_{N}\left(x_{j}\right)} \frac{\left(1-x^{2}\right) \dot{P}_{N}(x)}{x-x_{j}} .
$$

The collocation points, $x_{0}(=-1), x_{1}, x_{2}, \ldots, x_{N}(=1)$, are determined by the roots of the first derivative of the $N$ th-order Legendre polynomial with respect to $x$, namely, $\dot{P}_{N}\left(x_{i}\right)=0$.

For the electronic structure calculation involving Coulomb interaction, the potential has a singularity at $r=0$ and long tail at large $r$. These problems can be overcome by using the LGPS method associated with an appropriate mapping technique. In this procedure, the singularity problem can be taken considerate care of by the nonuniform collocation points of the LGPS method and the long-tail problem can be suppressed efficiently by mapping the semi-infinite domain $r \in[0, \infty]$ into the finite domain $x \in[-1,1]$ with a mapping function $r=r(x)$. The mapping function is taken to be a nonlinear function given by [26]

$$
r(x)=L \frac{1+x}{1-x+x_{m}},
$$

where $L$ is a mapping parameter used to optimize calculation by adjusting distribution of the collocation points and $x_{m}$ $=2 L / r_{\max }$, where $r_{\max }$ is the maximum $r$. This method provides a very effective and efficient numerical algorithm for a high-precision solution of the Schrödinger equation and the KS equation. It has been successfully applied to the calculation of eigenvalues and eigenfunctions of atomic and molecular systems for the study of electronic structure $[10,18]$, multiphoton processes in strong fields [31,32], and Rydberg atom spectroscopy and dynamics [33].

Making use of the mapping function (31) in the KS equation (16), we obtain a differential equation for the transformed radial wave function defined on $x \in[-1,1], f(x)$ $=R_{n l \sigma}(r(x)) / \sqrt{\dot{r}(x)}$, where $\dot{r}(x)$ is the first derivative of $r$ with respect to $x$. Applying the LGPS method to the new differential equation, considering boundary conditions, and following the symmetrization procedure [26], we finally 
achieve a symmetric matrix eigenvalue equation

$$
\sum_{j=1}^{N-1}\left[-\frac{1}{2} D_{i j}+u_{i} \delta_{i j}\right] \chi_{j}=\varepsilon_{n l \sigma} \chi_{i},
$$

where

$$
\begin{gathered}
\chi_{i}=\frac{\sqrt{\dot{r}_{i}}}{P_{N}\left(x_{i}\right)} R_{n l \sigma}\left(r_{i}\right), \\
u_{i}=\frac{l(l+1)}{2 r_{i}^{2}}+v_{\sigma}^{\mathrm{eff}}\left(r_{i}\right), \\
D_{i j}= \begin{cases}-\frac{2}{\dot{r}_{i}\left(x_{i}-x_{j}\right)^{2} \dot{r}_{j}}, & i \neq j, \\
-\frac{N(N+1)}{3 \dot{r}_{i}^{2}\left(1-x_{i}^{2}\right)}, & i=j,\end{cases}
\end{gathered}
$$

and $\chi_{i}=\chi\left(x_{i}\right), u_{i}=u\left(x_{i}\right), r_{i}=r\left(x_{i}\right)$, and $\dot{r}_{i}=\dot{r}\left(x_{i}\right)$. Solving the matrix eigenvalue equation (32), one can obtain the spin orbitals and orbital energies.

\section{Multiplet excited-state energy}

For a particular electron configuration, Eq. (32) is solved self-consistently to obtain a set of spin orbitals. Using these spin orbitals, a single Slater determinant for a specific electronic state is constructed and its total energy calculated. The total energy is a sum of noninteracting kinetic energy $E_{k}$, external-field energy $E_{\text {ext }}$, Hartree energy $E_{H}$, exchange energy $E_{x}$, and correlation energy $E_{c}$. They are evaluated by

$$
\begin{gathered}
E_{k}=\sum_{\sigma=\alpha}^{\beta} \sum_{i=1}^{N_{\sigma}} w_{i \sigma} \int \varphi_{i \sigma}(\mathbf{r})\left(-\frac{\nabla^{2}}{2}\right) \varphi_{i \sigma}(\mathbf{r}) d \mathbf{r} \\
=\sum_{\sigma=\alpha}^{\beta} \sum_{n l}^{\nu_{\sigma}} w_{n l \sigma} \int R_{n l \sigma}(r)\left(-\frac{1}{2} \frac{d^{2}}{d r^{2}}+\frac{l(l+1)}{2 r^{2}}\right) R_{n l \sigma}(r) d r \\
E_{e x t}=\int V_{e x t}(\mathbf{r}) \rho(\mathbf{r}) d \mathbf{r}=4 \pi \int v_{e x t}(r) \rho(r) r^{2} d r \\
E_{H}=\frac{1}{2} \int V_{H}(\mathbf{r}) \rho(\mathbf{r}) d \mathbf{r}=\frac{1}{2} \sum_{\Pi} \eta_{l m, l^{\prime} m^{\prime}}^{k} F_{n l \sigma, n^{\prime} l^{\prime} \sigma^{\prime}}^{k}, \\
E_{x=\alpha}^{\beta} \int V_{x \sigma}^{S}(\mathbf{r}) \rho_{\sigma}(\mathbf{r}) d \mathbf{r}=-\frac{1}{2} \sum_{\Pi} \lambda_{l m, l^{\prime} m^{\prime}}^{k} G_{n l \sigma, n^{\prime} l^{\prime} \sigma^{\prime}}^{k} \delta_{\sigma \sigma^{\prime}},
\end{gathered}
$$

and $E_{c}$ is computed by using the LYP approximation [7]. In Eqs. (38) and (39),

$$
\sum_{\Pi}=\sum_{\sigma, \sigma^{\prime}=\alpha}^{\beta} \sum_{n l m}^{\nu_{\sigma}} \sum_{n^{\prime} l^{\prime} m^{\prime}}^{\nu_{\sigma^{\prime}}} \sum_{k},
$$

$$
\eta_{l m, l^{\prime} m^{\prime}}^{k}=\langle l m k 0 \mid l m\rangle\left\langle l^{\prime} m^{\prime} k 0 \mid l^{\prime} m^{\prime}\right\rangle\langle l 0 k 0 \mid l 0\rangle\left\langle l^{\prime} 0 k 0 \mid l^{\prime} 0\right\rangle,
$$

$$
F_{n l \sigma, n^{\prime} l^{\prime} \sigma^{\prime}}^{k}=\int p_{n l \sigma, n l \sigma}(r) q_{n^{\prime} l^{\prime} \sigma^{\prime}, n^{\prime} l^{\prime} \sigma^{\prime}}^{k}(r) r^{2} d r,
$$

and

$$
G_{n l \sigma, n^{\prime} l^{\prime} \sigma^{\prime}}^{k}=\int p_{n l \sigma, n^{\prime} l^{\prime} \sigma^{\prime}}(r) q_{n l \sigma, n^{\prime} l^{\prime} \sigma^{\prime}}^{k}(r) r^{2} d r,
$$

where $k=0,2, \ldots, 2 \times \min \left(l, l^{\prime}\right)$ due to the Clebsch-Gordan coefficient.

From Eqs. (27) and (41)-(43), if $n^{\prime} l^{\prime} m^{\prime} \sigma^{\prime}=n l m \sigma$, the terms of $E_{H}$ in Eq. (38) completely cancel those of $E_{x}$ in Eq. (39). This illustrates that the X-only calculation presented here is free of self-interaction.

The procedure above can only be used to calculate the energy value for a single Slater determinant. For a multiplet state that can be described completely by a single Slater determinant, this energy is just the multiplet energy. For a multiplet state that cannot be represented by a single determinant, the energy can be calculated by means of Slater's diagonal sum rule [25]. According to this rule, the sum of the single-Slater-determinant energy $E\left(D_{i}\right)$ of determinant $D_{i}$ from an electron configuration is equal to the weighted sum of the multiplet energy $E\left(M_{j}\right)$ of the multiplet state $M_{j}$ involved in the electron configuration, namely,

$$
\sum_{i} E\left(D_{i}\right)=\sum_{j} d_{j} E\left(M_{j}\right)
$$

where the weight $d_{j}$ is the times that the multiplet state $M_{j}$ appears in all the single Slater determinants.

To show how to use this method, let us consider a twoelectron configuration $p^{2}$. For this configuration, there are 15 different states and thus 15 determinants corresponding to various values of $\Sigma m_{l}$ and $\Sigma m_{s}$. Of those, there are three determinants with $\left(m_{l_{1}} m_{s_{1}} ; m_{l_{2}} m_{s_{2}}\right)=(1 \alpha ;-1 \beta),(-1 \alpha ; 1 \beta)$, and $(0 \alpha ; 0 \beta)$ that correspond to a particular set of $\sum m_{l}=0$ and $\sum m_{s}=0$, which are denoted by $D_{1}, D_{2}$, and $D_{3}$, respectively. On the other hand, there are three multiplet states ${ }^{1} D$, ${ }^{3} P$, and ${ }^{1} S$ that are involved in this particular set, and each emerges once. According to Eq. (44) we have

$$
E\left(D_{1}\right)+E\left(D_{2}\right)+E\left(D_{3}\right)=E\left({ }^{1} D\right)+E\left({ }^{3} P\right)+E\left({ }^{1} S\right) .
$$

The energy terms on the left-hand side are computed by using the single Slater determinants, while the energies $E\left({ }^{1} D\right)$ and $E\left({ }^{3} P\right)$ on the right-hand side can also be determined by the single Slater determinants. Thus $E\left({ }^{1} S\right)$ is calculated from Eq. (45). Similar procedures have been employed in recent excited-state calculations $[18,19,34]$.

\section{RESULTS AND DISCUSSION}

\section{A. The ground-state energy}

We first calculate the total ground-state energies for the neutral atoms of $Z \leqslant 18$ using the procedure introduced in the 
TABLE I. Negative values of the total ground-state energies (a.u.) for neutral atoms of $Z \leqslant 18$.

\begin{tabular}{|c|c|c|c|c|c|}
\hline \multirow[b]{2}{*}{ Atoms } & \multicolumn{3}{|c|}{ X-only } & \multicolumn{2}{|c|}{$\mathrm{XC}$} \\
\hline & $\mathrm{SLHF}^{\mathrm{a}}$ & $\mathrm{HF}^{\mathrm{b}}$ & $\mathrm{OEP}^{\mathrm{c}}$ & $\mathrm{SLHF}^{\mathrm{a}}$ & $\mathrm{HFC}^{\mathrm{d}}$ \\
\hline $\mathrm{He}$ & 2.8617 & 2.8617 & & 2.9074 & 2.904 \\
\hline $\mathrm{Li}$ & 7.4325 & 7.4327 & 7.4325 & 7.4872 & 7.479 \\
\hline $\mathrm{Be}$ & 14.5726 & 14.5730 & 14.5724 & 14.6686 & 14.667 \\
\hline B & 24.5284 & 24.5291 & 24.5283 & 24.6586 & 24.654 \\
\hline $\mathrm{C}$ & 37.6886 & 37.6886 & 37.6889 & 37.8522 & 37.845 \\
\hline $\mathrm{N}$ & 54.4029 & 54.4009 & 54.4034 & 54.5977 & 54.590 \\
\hline $\mathrm{O}$ & 74.8115 & 74.8094 & 74.8121 & 75.0798 & 75.067 \\
\hline $\mathrm{F}$ & 99.4085 & 99.4093 & 99.4092 & 99.7393 & 99.731 \\
\hline $\mathrm{Ne}$ & 128.5455 & 128.5471 & 128.5454 & 128.9331 & 128.937 \\
\hline $\mathrm{Na}$ & 161.8565 & 161.8589 & 161.8566 & 162.2687 & 162.257 \\
\hline $\mathrm{Mg}$ & 199.6112 & 199.6146 & 199.6116 & 200.0744 & 200.059 \\
\hline $\mathrm{Al}$ & 241.8728 & 241.8767 & 241.8733 & 242.3726 & 242.356 \\
\hline $\mathrm{Si}$ & 288.8501 & 288.8544 & 288.8507 & 289.3853 & 289.374 \\
\hline $\mathrm{P}$ & 340.7140 & 340.7188 & 340.7150 & 341.2835 & 341.272 \\
\hline$S$ & 397.5018 & 397.5049 & 397.5016 & 398.1388 & 398.139 \\
\hline $\mathrm{Cl}$ & 459.4770 & 459.4821 & 459.4776 & 460.1750 & 460.196 \\
\hline $\mathrm{Ar}$ & 526.8118 & 526.8175 & 526.8122 & 527.5658 & 527.604 \\
\hline
\end{tabular}

${ }^{\mathrm{a} C u r r e n t}$ results.

${ }^{\mathrm{b}}$ Reference [35].

${ }^{\mathrm{c}}$ Reference [28].

${ }^{\mathrm{d}}$ References $[39,40]$.

preceding section. Special attention is paid to the nonsymmetric states of atoms with open shells. In Table I, we present the total ground-state energies obtained from X-only calculation together with those of the HF method [35] and the optimized effective potential (OEP) method [28] for comparison. Although the spherically averaged electronic density is used in the calculation, the present SLHF results are in excellent agreement with HF values even for the nonsymmetric states of the open-shell atoms. The maximum deviation to $\mathrm{HF}$ results in energy is about $0.0037 \%$. It occurs at a highly nonsymmetric state of $\mathrm{N}$, where the OEP energy also has the maximum deviation with respect to the HF value. The discrepancies of SLHF energies to OEP results are less than $0.0009 \%$, a negligibly small number. Thus SLHF has practically the same accuracy as OEP and is very close to HF.

To examine and appraise the SLHF exchange potential we give, in Table II, the exchange energies for the ground states of neutral atoms of $Z \leqslant 18$. We also list in this table the results of the HF method [6] and Becke's gradient-corrected density-functional exchange-energy approximation (BX) [36]. The overall agreement of our calculated exchange energies with the results of $\mathrm{HF}$ and $\mathrm{BX}$ is quite good. This indicates that the SLHF exchange potential has behaviors as good as those of the HF method.

Orbital energy is a measurement of the basic properties of electron orbital. The highest-occupied-orbital energy is even essential since it is related to the ionization potential in independent particle calculation such as the DFT calculation.
TABLE II. Negative values of the exchange energies (a.u.) for the ground states of neutral atoms of $Z \leqslant 18$.

\begin{tabular}{cccc}
\hline \hline Atoms & SLHF $^{\mathrm{a}}$ & $\mathrm{HF}^{\mathrm{b}}$ & $\mathrm{BX}^{\mathrm{c}}$ \\
\hline $\mathrm{He}$ & 1.026 & 1.026 & 1.025 \\
$\mathrm{Li}$ & 1.779 & 1.781 & 1.775 \\
$\mathrm{Be}$ & 2.663 & 2.667 & 2.658 \\
$\mathrm{~B}$ & 3.744 & 3.744 & 3.728 \\
$\mathrm{C}$ & 5.048 & 5.045 & 5.032 \\
$\mathrm{~N}$ & 6.599 & 6.596 & 6.589 \\
$\mathrm{O}$ & 8.181 & 8.174 & 8.169 \\
$\mathrm{~F}$ & 10.005 & 10.00 & 10.02 \\
$\mathrm{Ne}$ & 12.112 & 12.11 & 12.14 \\
$\mathrm{Na}$ & 14.023 & 14.02 & 14.03 \\
$\mathrm{Mg}$ & 15.986 & 15.99 & 16.00 \\
$\mathrm{Al}$ & 18.058 & 18.07 & 18.06 \\
$\mathrm{Si}$ & 20.262 & 20.28 & 20.27 \\
$\mathrm{P}$ & 22.618 & 22.64 & 22.62 \\
$\mathrm{~S}$ & 24.983 & 25.00 & 24.98 \\
$\mathrm{Cl}$ & 27.490 & 27.51 & 27.49 \\
$\mathrm{Ar}$ & 30.155 & 30.19 & 30.15 \\
\hline \hline
\end{tabular}

${ }^{\mathrm{a}}$ Current results.

${ }^{\mathrm{b}}$ Reference [6].

${ }^{\mathrm{c}}$ Reference [36].

TABLE III. Negative values of the highest-occupied-orbital energies (a.u.) for neutral atoms of $Z \leqslant 18$.

\begin{tabular}{ccccc}
\hline \hline Atoms & SLHF $^{\mathrm{a}}$ & HF $^{\mathrm{b}}$ & OEP $^{\mathrm{c}}$ & Expt. $^{\mathrm{d}}$ \\
\hline $\mathrm{He}$ & 0.9179 & 0.9180 & & 0.904 \\
$\mathrm{Li}$ & 0.1963 & 0.1963 & 0.1963 & 0.198 \\
$\mathrm{Be}$ & 0.3091 & 0.3093 & 0.3093 & 0.343 \\
$\mathrm{~B}$ & 0.3074 & 0.3099 & 0.3097 & 0.305 \\
$\mathrm{C}$ & 0.4250 & 0.4333 & 0.4353 & 0.414 \\
$\mathrm{~N}$ & 0.5707 & 0.5676 & 0.5712 & 0.534 \\
$\mathrm{O}$ & 0.5200 & 0.6319 & 0.5077 & 0.501 \\
$\mathrm{~F}$ & 0.6578 & 0.7300 & 0.6735 & 0.640 \\
$\mathrm{Ne}$ & 0.8495 & 0.8504 & 0.8507 & 0.793 \\
$\mathrm{Na}$ & 0.1821 & 0.1821 & 0.1821 & 0.189 \\
$\mathrm{Mg}$ & 0.2526 & 0.2531 & 0.2531 & 0.281 \\
$\mathrm{Al}$ & 0.2141 & 0.2100 & & 0.217 \\
$\mathrm{Si}$ & 0.2894 & 0.2971 & & 0.300 \\
$\mathrm{P}$ & 0.3911 & 0.3917 & & 0.385 \\
$\mathrm{~S}$ & 0.3719 & 0.4374 & & 0.381 \\
$\mathrm{Cl}$ & 0.4629 & 0.5064 & & 0.477 \\
$\mathrm{Ar}$ & 0.5902 & 0.5910 & 0.5908 & 0.579 \\
\hline \hline
\end{tabular}

${ }^{\mathrm{a} C u r r e n t}$ results.

${ }^{\mathrm{b}}$ Reference [35].

${ }^{\mathrm{c}}$ Reference [27].

${ }^{\mathrm{d}}$ References [37,38]. 
In Table III, we present the highest-occupied-orbital energies obtained from the X-only SLHF calculation for the ground states of atoms with $Z \leqslant 18$ along with experimental ionization potentials [37,38], HF results [35], and the results from spin-dependent OEP [27]. It is shown that our results are very close to OEP results. Compared to the experimental results, our results are even more accurate than HF results.

One of the most touchy effects in the DFT calculation is the correlation effect. This effect is considered through the correlation energy. So far, all the correlation functionals used in the DFT calculation are developed for the ground state. In this work, we choose the widely used LYP correlation energy functional [7] to estimate the electron correlation effect. In Table I, we present the total ground-state energies from the $\mathrm{XC}$ calculation for atoms with $Z \leqslant 18$. For comparison, we also list in Table I the total energies that are obtained from the sum of HF energies [39] and correlation energies [40] (HFC). It is shown that inclusion of the correlation effect does lead to a significant improvement in the ground-state energies compared to the HFC results. For the total energy, our results agree very well with HFC results. For larger atoms $(Z>8)$, deviations of our results to HFC results are less than $0.008 \%$. For smaller atoms $(Z \leqslant 8)$, our total energies are a little bit more negative than HFC results. The maximum discrepancy is about $0.12 \%$. The larger deviations for the smaller atoms are caused by the overestimated correlation energies computed with LYP correlation energy functional [7].

\section{B. Singly excited state}

The main purpose of this paper is to develop a procedure for the excited-state calculation of an atomic system, particularly for multiply excited states such as triply excited states. Before performing a multiply excited-state calculation, we would like to report some results for singly and doubly excited states of atomic systems so as to calibrate the accuracy of the approach presented in this paper.

As has been shown above, the total ground-state energies from an X-only calculation are quite accurate and comparable to HF results. Since the SLHF exchange potential is free of self-interactions, dependent on symmetries of electronic state, and has good long-range behaviors, it is expected to work well in the excited-state calculation of atomic system. In Table IV, we list the total energies from the XC calculation for singly excited states $1 s n s^{3} S$ and ${ }^{1} S$ $(n=1-16)$ of He together with the total energies of the complex rotation approach (CRA) [41] and the WF method (WF-I) [18]. According to the LYP correlation energy functional [7], the spin-dependent correlation interaction only occurs between electrons with different spins. For the triplet $S$ states in Table IV, correlation energies are zero and total energies from the X-only calculation are the same as those from the XC calculation. This may tempt one to conclude that the correlation energies for these states are underestimated by the LYP approximation, particularly for low excited states where the correlation interaction is expected to be larger than highly excited states. However, for the $1 s 2 s^{3} S$ state, our result, 2.17390 a.u., is only $0.06 \%$ higher than the
TABLE IV. Negative values of the total energies (a.u.) for singly excited states of He. The SLHF results are obtained from the XC calculation.

\begin{tabular}{ccccccc}
\hline \hline & \multicolumn{3}{c}{${ }^{3} S$} & & & \multicolumn{2}{c}{${ }^{1} S$} \\
\cline { 2 - 3 } \cline { 6 - 7 } Configuration & SLHF $^{\mathrm{a}}$ & CRA $^{\mathrm{b}}$ & WF-I $^{\mathrm{c}}$ & & SLHF $^{\mathrm{a}}$ & CRA $^{\mathrm{b}}$ \\
\hline $1 s 2 s$ & 2.17390 & 2.17523 & 2.17545 & 2.14471 & 2.14597 \\
$1 s 3 s$ & 2.06827 & 2.06869 & 2.06890 & 2.06226 & 2.06127 \\
$1 s 4 s$ & 2.03631 & 2.03651 & 2.03671 & 2.03423 & 2.03359 \\
$1 s 5 s$ & 2.02250 & 2.02262 & 2.02264 & 2.02157 & 2.02118 \\
$1 s 6 s$ & 2.01530 & 2.01538 & 2.01539 & 2.01482 & 2.01456 \\
$1 s 7 s$ & 2.01108 & 2.01113 & 2.01115 & 2.01080 & 2.01063 \\
$1 s 8 s$ & 2.00839 & 2.00843 & 2.00845 & 2.00821 & 2.00809 \\
$1 s 9 s$ & 2.00657 & 2.00660 & 2.00660 & 2.00646 & 2.00637 \\
$1 s 10 s$ & 2.00529 & 2.00531 & 2.00531 & & 2.00521 & 2.00514 \\
$1 s 11 s$ & 2.00435 & 2.00431 & 2.00431 & & 2.00429 & 2.00424 \\
$1 s 12 s$ & 2.00364 & 2.00365 & 2.00365 & 2.00359 & 2.00355 \\
$1 s 13 s$ & 2.00309 & 2.00310 & 2.00310 & 2.00306 & 2.00302 \\
$1 s 14 s$ & 2.00265 & 2.00266 & 2.00266 & 2.00263 & 2.00260 \\
$1 s 15 s$ & 2.00230 & 2.00231 & 2.00231 & 2.00228 & 2.00226 \\
$1 s 16 s$ & 2.00202 & & & 2.00203 & 2.00200 & 2.00199 \\
\hline
\end{tabular}

${ }^{\mathrm{a}}$ Current results.

${ }^{\mathrm{b}}$ Reference [41].

${ }^{c}$ Reference [18].

CRA result, 2.17523 a.u., and $0.07 \%$ higher than the WF-I result, 2.17545 a.u., indicating that our calculation is accurate and the correlation interaction in the triplet $S$ states is indeed small and plays an insignificant role. For the $1 s 2 s{ }^{1} S$ state, the deviation of our result with respect to the CRA result is $0.06 \%$, illustrating that the calculation for triplet $S$ states is as precise as that for singlet $S$ states. For a highly excited Rydberg state with large $n$, the agreement of our results with those of a more sophisticated CRA calculation becomes excellent for both multiplet states. This signifies that the SLHF exchange potential has very similar asymptotic long-range behaviors to that of CRA because the electronic structure of the highly excited Rydberg state is dominated by the asymptotic long-range Coulomb potential arising from exchange potential. For low excited states with small $n$, our results are slightly higher than those of CRA because of an underestimation of correlation energy. In all cases for both multiplet states, the deviations are less than $0.07 \%$. This demonstrates that the calculation with the SLHF exchange potential for singly excited states is comparable to the more sophisticated $a b$ initio calculation.

It should be mentioned that many quantum mechanical calculations confront a self-consistent convergence problem for highly excited states with large $n$ [42]. Even the WF method often encounters the same problem [19]. However, in our calculation, the advantages of the LGPS procedure over the Coulomb potential enable us to obtain converged results for all states by using the same number of grid points. It is straightforward to extend the calculation to even higher states, reflecting the effectiveness of the SLHF exchange potential for the excited-state calculation. 
TABLE V. Negative values of the total energies (a.u.) obtained from the XC calculation for singly excited states of He-like ions.

\begin{tabular}{|c|c|c|c|c|c|c|c|c|}
\hline \multirow[b]{2}{*}{ States } & \multicolumn{8}{|c|}{$Z$} \\
\hline & 3 & 4 & 5 & 6 & 7 & 8 & 9 & 10 \\
\hline \multirow[t]{2}{*}{$1 s 2 s^{3} S$} & 5.1089 & 9.2951 & 14.7317 & 21.4185 & 29.3554 & 38.5423 & 48.9792 & 60.6662 \\
\hline & $5.1043^{\mathrm{a}}$ & $9.2886^{\mathrm{a}}$ & $14.7240^{\mathrm{a}}$ & $21.4100^{\mathrm{a}}$ & & & & \\
\hline \multirow[t]{2}{*}{$1 s 2 s{ }^{1} S$} & 5.0463 & 9.1969 & 14.5959 & 21.2437 & 29.1406 & 38.2867 & 48.6826 & 60.3278 \\
\hline & $5.0422^{\mathrm{a}}$ & $9.1813^{\mathrm{a}}$ & $14.5710^{\mathrm{a}}$ & $21.2115^{\mathrm{a}}$ & & & & \\
\hline \multirow[t]{2}{*}{$1 s 2 p^{3} P$} & 5.0241 & 9.1708 & 14.5686 & 21.2170 & 29.1157 & 38.2645 & 48.6634 & 60.3124 \\
\hline & $5.0210^{\mathrm{a}}$ & $9.1647^{\mathrm{a}}$ & $14.5605^{\mathrm{a}}$ & $21.2075^{\mathrm{a}}$ & & & & \\
\hline \multirow[t]{2}{*}{$1 s 2 p{ }^{1} P$} & 5.0138 & 9.1394 & 14.5118 & 21.1321 & 29.0013 & 38.1196 & 48.4873 & 60.1045 \\
\hline & $4.9809^{\mathrm{a}}$ & $9.0886^{\mathrm{a}}$ & $14.4478^{\mathrm{a}}$ & $21.0587^{\mathrm{a}}$ & & & & \\
\hline
\end{tabular}

${ }^{\mathrm{a}}$ TDM results [43].

To check the feasibility of the SLHF exchange potential for atomic ions, we also apply it to the calculation of He-like ions $(Z=3-10)$. The total energies obtained from the XC calculation are shown in Table $\mathrm{V}$ together with the results of the truncated diagonalization method (TDM) [43]. It is shown that for all the data in the table, our results are close to the results of TDM. The deviations for the triplet $S$ and $P$

TABLE VI. Negative values of the total energies (a.u.) for singly excited states of Li.

\begin{tabular}{|c|c|c|c|c|c|}
\hline \multirow[b]{2}{*}{ States } & \multicolumn{2}{|c|}{ X-only } & \multicolumn{2}{|c|}{$\mathrm{XC}$} & \multirow{2}{*}{$\begin{array}{l}\text { Others } \\
\mathrm{CIH}^{\mathrm{c}}\end{array}$} \\
\hline & $\mathrm{SLHF}^{\mathrm{a}}$ & WF-I ${ }^{b}$ & $\mathrm{SLHF}^{\mathrm{a}}$ & $W F-I^{b}$ & \\
\hline $1 s^{2} 3 s^{2} S$ & 7.31003 & $\begin{array}{c}7.30966 \\
7.31021^{\mathrm{d}}\end{array}$ & 7.35953 & 7.35773 & 7.35410 \\
\hline $1 s^{2} 4 s^{2} S$ & 7.27478 & 7.27466 & 7.32316 & 7.31978 & 7.31853 \\
\hline $1 s^{2} 5 s^{2} S$ & 7.25991 & 7.25996 & 7.30785 & 7.30466 & 7.30355 \\
\hline $1 s^{2} 6 s^{2} S$ & 7.25227 & & 7.30000 & & \\
\hline $1 s^{2} 7 s^{2} S$ & 7.24783 & & 7.29546 & & \\
\hline $1 s^{2} 8 s^{2} S$ & 7.24502 & & 7.29259 & & \\
\hline $1 s^{2} 9 s^{2} S$ & 7.24313 & & 7.29067 & & \\
\hline $1 s^{2} 10 s^{2} S$ & 7.24180 & & 7.28932 & & \\
\hline $1 s^{2} 2 p^{2} P$ & 7.36474 & $\begin{array}{c}7.36486 \\
7.36507^{\mathrm{d}}\end{array}$ & 7.41683 & 7.41204 & 7.41016 \\
\hline $1 s^{2} 3 p^{2} P$ & 7.29296 & $\begin{array}{c}7.29295 \\
7.29319^{\mathrm{d}}\end{array}$ & 7.34236 & 7.33862 & 7.33716 \\
\hline $1 s^{2} 4 p^{2} P$ & 7.26804 & 7.26859 & 7.31646 & 7.31262 & 7.31190 \\
\hline $1 s^{2} 5 p^{2} P$ & 7.25658 & 7.25756 & 7.30457 & 7.30053 & 7.30030 \\
\hline $1 s^{2} 6 p^{2} P$ & 7.25038 & & 7.29816 & & \\
\hline $1 s^{2} 7 p^{2} P$ & 7.24665 & & 7.29432 & & \\
\hline $1 s^{2} 8 p^{2} P$ & 7.24424 & & 7.29184 & & \\
\hline $1 s^{2} 9 p^{2} P$ & 7.24258 & & 7.29013 & & \\
\hline $1 s^{2} 10 p^{2} P$ & 7.24140 & & 7.28893 & & \\
\hline
\end{tabular}

${ }^{\mathrm{a} C u r r e n t}$ results.

${ }^{\mathrm{b}}$ Reference [18].

${ }^{\mathrm{c}}$ Reference [45].

${ }^{\mathrm{d}}$ HF results [44]. states (where the correlation energies are zero) are less than $0.09 \%$, while for the singlet $S$ and $P$ states, the discrepancies are no more than $0.66 \%$ though a little bit larger. It demonstrates that the SLHF exchange potential together with the LYP correlation potential is reasonably accurate for the calculation of atomic ions. On the other hand, for all the data in the table, our results are a little bit more negative than those of TDM. This again hints that the LYP energy functional is overestimated to the correlation energies for smaller atoms as has already been pointed out in the calculations of neutral atoms.

TABLE VII. Negative values of the total energies (a.u.) for doubly excited states $n s^{2}{ }^{1} S(n=2-20)$ of He.

\begin{tabular}{|c|c|c|c|c|c|}
\hline \multirow[b]{2}{*}{ States } & \multicolumn{2}{|c|}{ X-only } & \multicolumn{3}{|c|}{$\mathrm{XC}$} \\
\hline & SLHF $^{\mathrm{a}}$ & WF-II ${ }^{b}$ & SLHF $^{\mathrm{a}}$ & WF-II ${ }^{b}$ & WF-I ${ }^{c}$ \\
\hline $2 s^{21} S$ & 0.71968 & 0.7197 & 0.73473 & 0.7333 & 0.76637 \\
\hline $3 s^{21} S$ & 0.31996 & 0.3200 & 0.33061 & 0.3265 & 0.34578 \\
\hline $4 s^{21} S$ & 0.17995 & 0.1800 & 0.18814 & 0.1838 & 0.19659 \\
\hline $5 s^{21} S$ & 0.11511 & 0.1152 & 0.12129 & 0.1177 & 0.12754 \\
\hline $6 s^{21} S$ & 0.07991 & 0.0800 & 0.08460 & 0.0818 & 0.08808 \\
\hline $7 s^{21} S$ & 0.05869 & 0.0588 & 0.06234 & 0.0601 & 0.06524 \\
\hline $8 s^{2}{ }^{1} S$ & 0.04492 & & 0.04783 & & 0.04855 \\
\hline $9 s^{2}{ }^{1} S$ & 0.03548 & & 0.03784 & & 0.03889 \\
\hline $10 s^{2}{ }^{1} S$ & 0.02873 & & 0.03068 & & 0.03084 \\
\hline $11 s^{21} S$ & 0.02374 & & 0.02538 & & 0.02503 \\
\hline $12 s^{21} S$ & 0.01994 & & 0.02134 & & 0.02121 \\
\hline $13 s^{21} S$ & 0.01698 & & 0.01819 & & 0.01811 \\
\hline $14 s^{21} S$ & 0.01464 & & 0.01569 & & 0.01555 \\
\hline $15 s^{21} S$ & 0.01275 & & 0.01367 & & 0.01348 \\
\hline $16 s^{21} S$ & 0.01120 & & 0.01202 & & 0.01176 \\
\hline $17 s^{21} S$ & 0.00992 & & 0.01064 & & 0.01132 \\
\hline $18 s^{21} S$ & 0.00885 & & 0.00949 & & \\
\hline $19 s^{2}{ }^{1} S$ & 0.00794 & & 0.00852 & & \\
\hline $20 s^{2}{ }^{1} S$ & 0.00716 & & 0.00769 & & \\
\hline
\end{tabular}

${ }^{\mathrm{a} C u r r e n t}$ results.

${ }^{\mathrm{b}}$ Reference [19].

${ }^{\mathrm{c}}$ Reference [18]. 
TABLE VIII. Negative values of the total energies (a.u.) for doubly excited states $n^{\prime} \operatorname{snp}{ }^{3} P\left(n^{\prime}=2-3, n=n^{\prime}-10\right)$ of He.

\begin{tabular}{|c|c|c|c|c|c|}
\hline \multirow[b]{2}{*}{ States } & \multirow{2}{*}{$\begin{array}{l}\text { X-only } \\
\text { WF-II }^{\text {b }}\end{array}$} & \multicolumn{2}{|c|}{$\mathrm{XC}$} & \multicolumn{2}{|c|}{ Other results } \\
\hline & & $\mathrm{SLHF}^{\mathrm{a}}$ & WF-I ${ }^{c}$ & $\mathrm{CCR}^{\mathrm{d}}$ & $\mathrm{RMSP}^{\mathrm{e}}$ \\
\hline $2 s 2 p^{3} P$ & 0.7537 & 0.75354 & $\begin{array}{c}0.76770 \\
0.7682^{b}\end{array}$ & 0.76049 & 0.76086 \\
\hline $2 s 3 p^{3} P$ & 0.5713 & 0.57193 & $\begin{array}{l}0.58253 \\
0.5804^{b}\end{array}$ & 0.58467 & 0.58476 \\
\hline $2 s 4 p^{3} P$ & & 0.53757 & 0.54558 & 0.54284 & 0.54281 \\
\hline $2 s 5 p^{3} P$ & & 0.52295 & 0.53121 & 0.52571 & 0.52475 \\
\hline $2 s 6 p^{3} P$ & & 0.51553 & 0.52367 & 0.51711 & \\
\hline $2 s 7 p^{3} P$ & & 0.51119 & 0.51924 & 0.51219 & \\
\hline $2 s 8 p^{3} P$ & & 0.50847 & & 0.50910 & \\
\hline $2 s 9 p^{3} P$ & & 0.50663 & & & \\
\hline $2 s 10 p^{3} P$ & & 0.50533 & & & \\
\hline $3 s 3 p^{3} P$ & 0.3417 & 0.34167 & $\begin{array}{l}0.34918 \\
0.3486^{\mathrm{b}}\end{array}$ & 0.35038 & 0.35082 \\
\hline $3 s 4 p^{3} P$ & & 0.27156 & 0.27365 & 0.27948 & 0.27980 \\
\hline $3 s 5 p^{3} P$ & & 0.24826 & & 0.25516 & 0.25509 \\
\hline $3 s 6 p^{3} P$ & & 0.23939 & & 0.24338 & 0.24274 \\
\hline $3 s 7 p^{3} P$ & & 0.23442 & & 0.23688 & \\
\hline $3 s 8 p^{3} P$ & & 0.23133 & & & \\
\hline $3 s 9 p^{3} P$ & & 0.22929 & & & \\
\hline $3 s 10 p^{3} P$ & & 0.22787 & & & \\
\hline
\end{tabular}

${ }^{\mathrm{a} C}$ Current results.

${ }^{b}$ WF-II results [19].

${ }^{\mathrm{c}} \mathrm{WF}-\mathrm{I}$ results [18].

${ }^{\mathrm{d}}$ Reference [47].

${ }^{\mathrm{e}}$ Reference [48].

In Table VI, we present the total energies obtained from both the X-only calculation and the XC calculation for singly excited states $1 s^{2} n s^{2} S(n=3-10)$ and $1 s^{2} n p{ }^{2} P(n=2-10)$ of Li. For comparison, we also list the total energies of the WF method (WF-I) [18], the HF method [44], and the configuration-interaction Hylleraas method (CIH) [45]. The deviations of $\mathrm{X}$-only results to $\mathrm{HF}$ results are $0.0025 \%$, $0.0045 \%$, and $0.0032 \%$ for $1 s^{2} 3 s^{2} S, 1 s^{2} 2 p^{2} P$, and $1 s^{2} 3 p^{2} P$, respectively. This illustrates that our X-only calculation is quite accurate, almost as accurate as the HF method. The deviations of X-only results to WF-I results range from $0.0051 \%$ to $0.0007 \%$ for $1 s^{2} n s^{2} S(n=3-5)$ states and from $0.0135 \%$ to $0.0001 \%$ for $1 s^{2} n p^{2} P(n=2-5)$ states. For the total energies obtained from the $\mathrm{XC}$ calculation, our results are only a little bit more negative than the WF results and $\mathrm{CIH}$ results due to the overestimation to the correlation energies by the LYP correlation energy functional. Nevertheless, the maximum discrepancy of our results to the $\mathrm{CIH}$ results is no more than $0.0738 \%$ for $1 s^{2} n s^{2} S(n=3-5)$ states and $0.0900 \%$ for $1 s^{2} n p^{2} P(n=2-5)$ states, demonstrating the accuracy of our calculation.
TABLE IX. Negative values of the total energies (a.u.) for doubly excited states $n^{\prime} s n p{ }^{1} P\left(n^{\prime}=1-2, n=n^{\prime}-10\right)$ of He.

\begin{tabular}{ccccc}
\hline \hline States & $\begin{array}{c}\text { X-only } \\
\text { SLHF }^{\mathrm{a}}\end{array}$ & $\begin{array}{c}\text { XC } \\
\text { SLHF }^{\mathrm{a}}\end{array}$ & $\begin{array}{c}\text { Other } \\
\text { theory }\end{array}$ & $\begin{array}{c}\text { Expt. } \\
\text { results }\end{array}$ \\
\hline $2 s 2 p{ }^{\mathrm{e} P}$ & 0.65039 & 0.69699 & $0.69275^{\mathrm{c}}$ & \\
& $0.6456^{\mathrm{b}}$ & $0.6588^{\mathrm{b}}$ & & \\
$2 s 3 p{ }^{1} P$ & 0.56650 & 0.57407 & $0.56384^{\mathrm{c}}$ & \\
$2 s 4 p{ }^{1} P$ & 0.53568 & 0.53860 & $0.53418^{\mathrm{c}}$ & \\
$2 s 5 p{ }^{1} P$ & 0.52225 & 0.52349 & & \\
$2 s 6 p{ }^{1} P$ & 0.51519 & 0.51587 & & \\
$2 s 7 p{ }^{1} P$ & 0.51104 & 0.51134 & & \\
$2 s 8 p{ }^{1} P$ & 0.50836 & 0.50852 & & \\
$2 s 9 p{ }^{1} P$ & 0.50656 & 0.50665 & & \\
$2 s 10 p{ }^{1} P$ & 0.50528 & 0.50533 & & \\
$3 s 3 p{ }^{1} P$ & 0.29101 & 0.33001 & $0.33566^{\mathrm{d}}$ & 0.33330 \\
$3 s 4 p{ }^{1} P$ & 0.26380 & 0.27009 & $0.27110^{\mathrm{d}}$ & 0.27014 \\
$3 s 5 p{ }^{1} P$ & 0.24730 & 0.25019 & $0.25058^{\mathrm{d}}$ & 0.25020 \\
$3 s 6 p{ }^{1} P$ & 0.23897 & 0.24053 & $0.24074^{\mathrm{d}}$ & 0.24060 \\
$3 s 7 p{ }^{1} P$ & 0.23417 & 0.23510 & $0.23574^{\mathrm{d}}$ & 0.23530 \\
$3 s 8 p{ }^{1} P$ & 0.23119 & 0.23178 & $0.23260^{\mathrm{d}}$ & 0.23184 \\
$3 s 9 p{ }^{1} P$ & 0.22919 & 0.22956 & & \\
$3 s 10 p{ }^{1} P$ & 0.22780 & 0.22807 & & \\
\hline \hline
\end{tabular}

${ }^{\mathrm{a} C u r r e n t}$ results.

${ }^{\mathrm{b}} \mathrm{WF}-\mathrm{II}$ results [19].

${ }^{\mathrm{c}} \mathrm{RMSP}$ results [48].

${ }^{\mathrm{d}}$ FF results [49].

${ }^{\mathrm{e}}$ Experimental results [50].

\section{Doubly excited state}

The calculation of doubly excited state is more challenging. For doubly excited states, the results of the HF method are rare. To check the applicability and calibrate the accuracy of the SLHF exchange potential in the doubly excited-state calculation, we will compare our X-only results with other $\mathrm{X}$-only DFT results available in the literature. In Tables VII-X, we present the total energies obtained from both the $\mathrm{X}$-only calculation and the XC calculation for doubly excited states $n s^{2}{ }^{1} S \quad(n=2-20), n^{\prime} \operatorname{snp}{ }^{3} P \quad\left(n^{\prime}=2-3, \quad n=n^{\prime}-10\right)$, $n^{\prime} \operatorname{snp}{ }^{1} P\left(n^{\prime}=2-3, n=n^{\prime}-10\right)$, and $n p^{2}{ }^{1} D(n=2-20)$, of $\mathrm{He}$, respectively. In these tables, we also list the available experimental results and other theoretical results for comparison.

In Table VII, we report the total energies for doubly excited Rydberg states $n s^{2}{ }^{1} S\left(n^{\prime}=2-20\right)$. For the X-only calculation, our results are in very good agreement with those of the WF method (WF-II) [19]. For the XC calculation, our results are a little bit more negative than those of WF-II and less negative than those from another WF calculation (WF-I) [18]. Obviously, the deviations are caused by correlation energies since the correlation energy functionals used in these calculations are different from one another. A Wigner-type parametrized correlation energy functional [46] was used in the WF-II, a spin-independent LYP correlation energy functional was used in the WF-I, and a spin-dependent LYP cor- 
TABLE X. Negative values of the total energies (a.u.) for doubly excited states $n p^{2}{ }^{1} D(n=2-20)$ of He.

\begin{tabular}{|c|c|c|c|c|c|}
\hline \multirow[b]{2}{*}{ States } & \multicolumn{2}{|c|}{ X-only } & \multicolumn{3}{|c|}{$\mathrm{XC}$} \\
\hline & SLHF $^{\mathrm{a}}$ & WF-II ${ }^{b}$ & SLHF $^{\mathrm{a}}$ & WF-II ${ }^{b}$ & WF-I ${ }^{\mathrm{c}}$ \\
\hline $2 p^{21} D$ & 0.66868 & 0.6679 & 0.69626 & 0.6823 & 0.69272 \\
\hline $3 p^{21} D$ & 0.30934 & 0.3091 & 0.32360 & 0.3160 & 0.31540 \\
\hline $4 p^{21} D$ & 0.17606 & 0.1760 & 0.18565 & 0.1800 & 0.18095 \\
\hline $5 p^{21} D$ & 0.11326 & 0.1132 & 0.12005 & 0.1158 & 0.11610 \\
\hline $6 p^{21} D$ & 0.07886 & 0.0788 & 0.08386 & 0.0807 & 0.08115 \\
\hline $7 p^{21} D$ & 0.05803 & 0.0580 & 0.06183 & 0.0593 & 0.05980 \\
\hline $8 p^{2}{ }^{1} D$ & 0.04447 & & 0.04745 & & 0.04565 \\
\hline $9 p^{2}{ }^{1} D$ & 0.03516 & & 0.03756 & & 0.03604 \\
\hline $10 p^{21} D$ & 0.02849 & & 0.03046 & & 0.02920 \\
\hline $11 p^{21} D$ & 0.02356 & & 0.02520 & & 0.02414 \\
\hline $12 p^{21} D$ & 0.01980 & & 0.02119 & & 0.02028 \\
\hline $13 p^{2}{ }^{1} D$ & 0.01687 & & 0.01807 & & 0.01728 \\
\hline $14 p^{21} D$ & 0.01455 & & 0.01559 & & 0.01490 \\
\hline $15 p^{2}{ }^{1} D$ & 0.01268 & & 0.01358 & & 0.01297 \\
\hline $16 p^{2}{ }^{1} D$ & 0.01114 & & 0.01194 & & 0.01140 \\
\hline $17 p^{2}{ }^{1} D$ & 0.00987 & & 0.01058 & & 0.01010 \\
\hline $18 p^{2}{ }^{1} D$ & 0.00881 & & 0.00944 & & \\
\hline $19 p^{2}{ }^{1} D$ & 0.00790 & & 0.00848 & & \\
\hline $20 p^{2}{ }^{1} D$ & 0.00713 & & 0.00765 & & \\
\hline
\end{tabular}

${ }^{\mathrm{a}}$ Current results.

${ }^{\mathrm{b}}$ Reference [19].

${ }^{\mathrm{c}}$ Reference [18]. relation energy functional is used in our calculation. Because both the Wigner-type correlation energy functional and the LYP correlation energy functional are designed basically for the ground-state calculation, all the calculations for the excited states by using these functionals are only rough estimations to the correlation energies.

In Table VIII, we present the results of doubly excited states $n^{\prime} \operatorname{snp}{ }^{3} P\left(n^{\prime}=2-3, n=n^{\prime}-10\right)$ of He. As has already been mentioned, the correlation energies obtained from the LYP correlation energy functional are zero for electrons with the same spin in the spin-dependent calculation. Thus the total energies from the $\mathrm{X}$-only calculation are the same as those from the $\mathrm{XC}$ calculation. For the X-only calculation, our results agree well with the WF-II results. For the $\mathrm{XC}$ calculation, our results are close to those of the WF-I, WF-II, the complex-coordinate rotation (CCR) methods [47], and the $R$-matrix method with saddle-point technique (RMSP) [48]. For $2 \operatorname{snp}{ }^{3} P(n=2-8)$ states, the deviations of our results to the results of the CCR method are less than $1.0 \%$ with an exception of state $2 s 3 p^{3} P$, for which the deviation is $2.2 \%$. For $3 \operatorname{snp}^{3} P(n=3-7)$ states, the errors are a little bit larger, but no more than $2.8 \%$. The larger deviations may again be induced by the underestimated (zero) correlation energies by the LYP correlation energy functional.

In Table IX we show the total energies of doubly excited states $n^{\prime} s n p{ }^{1} P\left(n^{\prime}=2-3, n=n^{\prime}-10\right)$ of He. For the X-only calculation, our result for $2 s 2 p{ }^{1} P$ is $0.74 \%$ lower than the WF-II result. For the XC calculation, our result for this state is better than the WF-II result compared to the result of the RMSP [48]. The deviations of our results to the RMSP results are less than $1.8 \%$ for states $2 s n{ }^{1} P(n=2-4)$. For

TABLE XI. Negative values of the total energies (a.u.) for triply excited states of Li-like ions $(Z=3-10)$.

\begin{tabular}{|c|c|c|c|c|c|c|c|c|}
\hline \multirow[b]{2}{*}{ States } & \multicolumn{3}{|c|}{$\mathrm{Li}$} & \multicolumn{3}{|c|}{$\mathrm{Be}^{+}$} & \multicolumn{2}{|c|}{$\mathrm{B}^{2+}$} \\
\hline & SLHF $^{\mathrm{a}}$ & $\mathrm{TDM}^{\mathrm{b}}$ & $\mathrm{MSPCR}^{\mathrm{c}}$ & $\mathrm{SLHF}^{\mathrm{a}}$ & $\mathrm{TDM}^{\mathrm{b}}$ & $\mathrm{MSPCR}^{\mathrm{d}}$ & $\mathrm{SLHF}^{\mathrm{a}}$ & $\mathrm{TDM}^{\mathrm{b}}$ \\
\hline $2 s 2 p^{24} P$ & 2.2209 & $2.2331(0.55)$ & $2.2394(0.83)$ & 4.4163 & $4.4248(0.19)$ & $4.4361(0.45)$ & 7.3617 & $7.3671(0.07)$ \\
\hline $2 s 2 p^{22} D$ & 2.1517 & $2.1480(0.17)$ & $2.1582(0.30)$ & 4.3052 & $4.2885(0.39)$ & $4.3073(0.05)$ & 7.2062 & $7.1792(0.38)$ \\
\hline $2 s 2 p^{2}{ }^{2} S$ & 2.0821 & $2.0827(0.03)$ & $2.0950(0.62)$ & 4.2038 & $4.1853(0.44)$ & $4.2085(0.11)$ & 7.0731 & $7.0415(0.45)$ \\
\hline $2 s 2 p^{22} P$ & 2.0960 & $2.0675(1.38)$ & $2.0788(0.83)$ & 4.2184 & $4.1711(1.13)$ & $4.1900(0.68)$ & 7.0861 & $7.0245(0.88)$ \\
\hline $2 p^{34} S$ & 2.0762 & $2.0953(0.91)$ & & 4.2085 & $4.2221(0.32)$ & & 7.0910 & $7.1000(0.13)$ \\
\hline $2 p^{32} D$ & 2.0606 & $2.0700(0.45)$ & $2.0790(0.89)$ & 4.1704 & $4.1655(0.12)$ & $4.1832(0.31)$ & 7.0275 & $7.0108(0.24)$ \\
\hline \multirow[t]{2}{*}{$2 p^{32} P$} & 2.0159 & $2.0035(0.62)$ & $2.0124(0.17)$ & 4.1046 & $4.0542(1.24)$ & $4.0755(0.71)$ & 6.9405 & 6.8611(1.16) \\
\hline & \multicolumn{3}{|c|}{$\mathrm{C}^{3+}$} & $4+$ & & $56+$ & & \\
\hline States & $\mathrm{SLHF}^{\mathrm{a}}$ & $\mathrm{TDM}^{\mathrm{b}}$ & $\mathrm{MSPCR}^{\mathrm{d}}$ & $\mathrm{SLHF}^{\mathrm{a}}$ & SLHF $^{\mathrm{a}}$ & $\mathrm{SLHF}^{\mathrm{a}}$ & $\mathrm{SLHF}^{\mathrm{a}}$ & \\
\hline $2 s 2 p^{2}{ }^{4} P$ & 11.0571 & $11.0601(0.03)$ & $11.0777(0.19)$ & 15.5025 & 20.6978 & 26.6432 & 33.3385 & \\
\hline $2 s 2 p^{2}{ }^{2} D$ & 10.8557 & $10.8207(0.32)$ & $10.8509(0.04)$ & 15.2541 & 20.4017 & 26.2989 & 32.9457 & \\
\hline $2 s 2 p^{22} S$ & 10.6908 & $10.6494(0.39)$ & $10.6860(0.04)$ & 15.0574 & 20.1734 & 26.0389 & 32.6540 & \\
\hline $2 s 2 p^{2}{ }^{2} P$ & 10.7010 & $10.6283(0.68)$ & $10.6597(0.39)$ & 15.0640 & 20.1758 & 26.0367 & 32.6469 & \\
\hline $2 p^{34} S$ & 10.7236 & $10.7289(0.05)$ & & 15.1063 & 20.2390 & 26.1218 & 32.7545 & \\
\hline $2 p^{32} D$ & 10.6329 & $10.6068(0.25)$ & $10.6382(0.05)$ & 14.9872 & 20.0909 & 25.9440 & 32.5468 & \\
\hline $2 p^{32} P$ & 10.5249 & $10.4195(1.01)$ & $10.4574(0.65)$ & 14.8582 & 19.9407 & 25.7728 & 32.3544 & \\
\hline
\end{tabular}

${ }^{\mathrm{a} C u r r e n t}$ results.

${ }^{\mathrm{b}}$ Reference [43].
${ }^{\mathrm{c}}$ Reference [52].

${ }^{\mathrm{d}}$ Reference [51]. 
TABLE XII. Autoionization channel energies (eV) for the triply excited resonance states of Li-like ions.

\begin{tabular}{|c|c|c|c|c|c|c|c|c|c|c|c|}
\hline \multirow{2}{*}{$\begin{array}{l}\text { Initial } \\
\text { states }\end{array}$} & \multirow{2}{*}{$\begin{array}{l}\text { Final } \\
\text { states }\end{array}$} & \multicolumn{5}{|c|}{$\mathrm{Li}$} & \multicolumn{5}{|c|}{$\mathrm{Be}^{+}$} \\
\hline & & $\mathrm{SLHF}^{\mathrm{a}}$ & $\mathrm{TDM}^{\mathrm{b}}$ & $\mathrm{MSPCR}^{\mathrm{c}}$ & \multicolumn{2}{|c|}{ Expt. $^{d}$} & $\mathrm{SLHF}^{\mathrm{a}}$ & $\mathrm{TDM}^{\mathrm{b}}$ & \multicolumn{2}{|c|}{$\mathrm{MSPCR}^{\mathrm{e}}$} & Expt. $^{d}$ \\
\hline $2 s 2 p^{2}{ }^{4} P$ & $1 s 2 p^{3} P$ & 76.280 & $75.864(0.55)$ & $75.879(0.53)$ & \multicolumn{2}{|c|}{$75.88 \pm 0.1(0.53)$} & 129.378 & $128.980(0.31)$ & \multicolumn{2}{|c|}{$128.992(0.30)$} & $128.9 \pm 0.2(0.37)$ \\
\hline \multirow[t]{4}{*}{$2 s 2 p^{22} D$} & $1 s 2 s^{3} S$ & 80.470 & $80.444(0.03)$ & $80.349(0.15)$ & \multicolumn{2}{|c|}{$80.26 \pm 0.1(0.26)$} & 135.783 & $136.061(0.20)$ & \multicolumn{2}{|c|}{$135.839(0.04)$} & $135.8 \pm 0.2(0.01)$ \\
\hline & $1 s 2 s{ }^{1} S$ & 78.767 & $78.755(0.02)$ & $78.447(0.41)$ & \multirow{2}{*}{\multicolumn{2}{|c|}{$78.13 \pm 0.1(0.04)$}} & 133.111 & $133.140(0.02)$ & \multicolumn{2}{|c|}{$132.783(0.25)$} & $132.8 \pm 0.2(0.23)$ \\
\hline & $1 s 2 p^{3} P$ & 78.163 & $78.178(0.02)$ & $78.089(0.09)$ & & & 132.401 & $132.688(0.22)$ & \multicolumn{2}{|c|}{$132.514(0.09)$} & $132.4 \pm 0.2(0.00)$ \\
\hline & $1 s 2 p^{1} P$ & 77.882 & $77.086(1.03)$ & $77.153(0.94)$ & \multicolumn{2}{|c|}{$78.13 \pm 0.1(0.04)$} & 131.546 & $130.618(0.71)$ & \multicolumn{2}{|c|}{$130.766(0.60)$} & \\
\hline \multirow[t]{4}{*}{$2 s 2 p^{2}{ }^{2} S$} & $1 s 2 s^{3} S$ & 82.364 & $82.220(0.18)$ & $82.067(0.36)$ & \multicolumn{2}{|c|}{$82.06 \pm 0.1(0.37)$} & 138.543 & $138.869(0.23)$ & \multicolumn{2}{|c|}{$138.532(0.01)$} & $138.5 \pm 0.3(0.03)$ \\
\hline & $1 s 2 s{ }^{1} S$ & 80.661 & $80.532(0.16)$ & $80.165(0.62)$ & \multicolumn{2}{|c|}{$80.26 \pm 0.1(0.50)$} & 135.870 & $135.948(0.06)$ & & $135.4 \pm 0.3(0.35)$ \\
\hline & $1 s 2 p^{3} P$ & 80.057 & $79.955(0.13)$ & $79.807(0.31)$ & \multicolumn{2}{|c|}{$* 79.9 \pm 0.1(0.20)$} & 135.160 & $135.496(0.25)$ & 135. & $07(0.03)$ & \\
\hline & $1 s 2 p^{1} P$ & 79.776 & $78.862(1.16)$ & $78.871(1.15)$ & \multicolumn{2}{|c|}{$* 78.7 \pm 0.2(1.37)$} & 134.306 & $133.426(0.66)$ & 133. & $60(0.63)$ & \\
\hline $2 s 2 p^{2}{ }^{2} P$ & $1 s 2 p^{3} P$ & 79.678 & $80.369(0.86)$ & $80.250(0.71)$ & $80.26 \pm 0.1$ & $.73)$ & 134.763 & $135.884(0.82)$ & 135. & $60(0.66)$ & $135.8 \pm 0.2(0.76)$ \\
\hline & $1 s 2 p^{1} P$ & 79.398 & $79.278(0.15)$ & $79.314(0.11)$ & $79.30 \pm 0.1$ & $.12)$ & 133.908 & $133.813(0.07)$ & 133. & $13(0.00)$ & $134.0 \pm 0.2(0.07)$ \\
\hline $2 p^{32} D$ & $1 s 2 p^{3} P$ & 80.642 & $80.301(0.42)$ & $80.244(0.50)$ & $80.26 \pm 0.1$ & $.48)$ & 136.069 & $136.036(0.02)$ & 135. & $79(0.14)$ & $135.8 \pm 0.2(0.20)$ \\
\hline & $1 s 2 p^{1} P$ & 80.361 & $79.210(1.45)$ & $79.308(1.33)$ & $79.30 \pm 0.1$ & $.34)$ & 135.214 & $133.965(0.93)$ & 134. & $32(0.81)$ & $134.0 \pm 0.2(0.91)$ \\
\hline $2 p^{32} P$ & $1 s 2 s^{3} S$ & 84.165 & $84.378(0.25)$ & $84.315(0.18)$ & & & 141.242 & $142.436(0.84)$ & 142. & $66(0.65)$ & $142.4 \pm 0.3(0.81)$ \\
\hline & $1 s 2 s{ }^{1} S$ & 82.462 & $82.688(0.27)$ & $82.413(0.06)$ & & & 138.570 & $139.517(0.68)$ & 139. & $10(0.39)$ & \\
\hline & $1 s 2 p^{3} P$ & 81.858 & $82.111(0.31)$ & $82.055(0.24)$ & $82.06 \pm 0.1$ & $.25)$ & 137.859 & $139.065(0.87)$ & 138. & $41(0.71)$ & $138.8 \pm 0.2(0.68)$ \\
\hline & $1 s 2 p^{1} P$ & 81.578 & $81.020(0.69)$ & $81.120(0.56)$ & $* 81.2 \pm 0.2$ & $.47)$ & 137.005 & $136.994(0.01)$ & 137. & $94(0.06)$ & $137.0 \pm 0.3(0.00)$ \\
\hline Initial & Final & & $\mathrm{B}^{2+}$ & & $3+$ & $\mathrm{N}^{4+}$ & $\mathrm{O}^{5+}$ & & $\mathrm{F}^{6+}$ & & $\mathrm{Ne}^{7+}$ \\
\hline states & states & SLHF $^{\mathrm{a}}$ & $\mathrm{TDM}^{\mathrm{b}}$ & $\mathrm{SLHF}^{\mathrm{a}}$ & $\mathrm{TDM}^{\mathrm{b}}$ & $\mathrm{SLHF}^{\mathrm{a}}$ & $\mathrm{SLHF}^{\mathrm{a}}$ & SLHF $^{\mathrm{a}}$ & $\mathrm{HSCC}^{\mathrm{f}}$ & Expt. $^{\mathrm{g}}$ & SLHF $^{\mathrm{a}}$ \\
\hline $2 s 2 p^{24} P$ & $1 s 2 p^{3} P$ & 196.111 & $195.743(0.19)$ & 276.467 & $276.126(0.12)$ & 370.437 & 478.018 & 599.205 & $599.29(0.01)$ & $599.0 \pm 0.7(0.03)$ & 734.003 \\
\hline $2 s 2 p^{2}{ }^{2} D$ & $1 s 2 s^{3} S$ & 204.781 & $205.306(0.26)$ & 287.431 & $288.153(0.25)$ & 383.719 & 493.635 & 617.167 & $618.39(0.20)$ & $619.1 \pm 0.8(0.31)$ & 754.319 \\
\hline & $1 s 2 s^{1} S$ & 201.086 & $201.141(0.03)$ & 282.674 & $282.751(0.03)$ & 377.874 & 486.679 & 609.096 & $609.35(0.04)$ & $609.4 \pm 0.5(0.05)$ & 745.111 \\
\hline & $1 s 2 p^{3} P$ & 200.343 & $200.856(0.26)$ & 281.948 & $282.642(0.25)$ & 377.196 & 486.075 & 608.574 & $609.74(0.19)$ & $609.4 \pm 0.5(0.14)$ & 744.692 \\
\hline & $1 s 2 p^{1} P$ & 198.797 & $197.791(0.51)$ & 279.637 & $278.592(0.38)$ & 374.083 & 482.132 & 603.782 & (20) & & 739.034 \\
\hline $2 s 2 p^{2}{ }^{2} S$ & $1 s 2 s^{3} S$ & 208.403 & $209.053(0.31)$ & 291.918 & $292.812(0.31)$ & 389.071 & 499.847 & 624.242 & $625.73(0.24)$ & $625.4 \pm 0.5(0.19)$ & 762.257 \\
\hline & $1 s 2 s^{1} S$ & 204.707 & $204.888(0.09)$ & 287.161 & $287.410(0.09)$ & 383.226 & 492.892 & 616.171 & $617.08(0.15)$ & $616.7 \pm 0.5(0.09)$ & 753.048 \\
\hline & $1 s 2 p^{3} P$ & 203.965 & $204.603(0.31)$ & 286.435 & $287.301(0.30)$ & 382.549 & 492.288 & 615.649 & $617.47(0.29)$ & $616.7 \pm 0.5(0.17)$ & 752.629 \\
\hline & $1 s 2 p^{1} P$ & 202.419 & $201.538(0.44)$ & 284.124 & $283.251(0.31)$ & 379.436 & 488.345 & 610.857 & & & 746.972 \\
\hline $2 s 2 p^{2}{ }^{2} P$ & $1 s 2 p^{3} P$ & 203.611 & $205.067(0.71)$ & 286.157 & $287.877(0.60)$ & 382.369 & 492.222 & 615.709 & 618.11(0.39) & $619.1 \pm 0.8(0.55)$ & 752.823 \\
\hline & $1 s 2 p^{1} P$ & 202.065 & $202.000(0.03)$ & 283.847 & $283.828(0.01)$ & 379.256 & 488.280 & 610.917 & $611.73(0.13)$ & $611.9 \pm 0.6(0.16)$ & 747.165 \\
\hline $2 p^{32} D$ & $1 s 2 p^{3} P$ & 205.205 & $205.439(0.11)$ & 288.010 & $288.462(0.16)$ & 384.459 & 494.533 & 618.231 & $619.04(0.13)$ & $619.1 \pm 0.8(0.14)$ & 755.546 \\
\hline & $1 s 2 p^{1} P$ & 203.660 & $202.373(0.64)$ & 285.700 & $284.413(0.45)$ & 381.346 & 490.590 & 613.439 & $613.60(0.03)$ & $613.6 \pm 0.6(0.03)$ & 749.889 \\
\hline $2 p^{32} P$ & $1 s 2 s{ }^{3} S$ & 212.011 & 213.962(0.91) & 296.432 & 299.069(0.88) & 394.492 & 506.179 & 631.483 & & & 770.409 \\
\hline & $1 s 2 s^{1} S$ & 208.316 & $209.799(0.71)$ & 291.676 & $293.668(0.68)$ & 388.647 & 499.224 & 623.412 & & & 761.201 \\
\hline & $1 s 2 p^{3} P$ & 207.573 & $209.513(0.93)$ & 290.949 & $293.559(0.89)$ & 387.969 & 498.620 & 622.890 & $627.12(0.67)$ & $627.1 \pm 0.5(0.67)$ & 760.782 \\
\hline & $1 s 2 p^{1} P$ & 206.027 & $206.446(0.20)$ & 288.639 & $289.510(0.30)$ & 384.856 & 494.677 & 618.098 & & & 755.125 \\
\hline${ }^{\mathrm{a}}$ Current $\mathrm{r}$ & & & & & & & nce $[51]$. & & & & \\
\hline${ }^{\mathrm{b}}$ Referenc & 3]. & & & & & & nce [54]. & & & & \\
\hline${ }^{\mathrm{c}}$ Referenc & $2]$. & & & & & & rimental $\mathrm{r}$ & ults [54]. & & & \\
\hline
\end{tabular}


$3 \operatorname{snp}{ }^{1} P(n=3-8)$ states, the discrepancies of our results are less than $1.7 \%$ with respect to the results of the Feshbach formalism approach (FF) [49] and less than $1.0 \%$ with respect to the experimental results [50], demonstrating the accuracy of our excited-state calculation.

In Table X, we report the total energies of doubly excited states $n p^{2}{ }^{1} D(n=2-10)$ of He. It is shown again that X-only results are in very good agreement with those of WF-II [19] and XC results are close to those of WF-I [18].

\section{Triply excited states}

For the DFT calculation, triply excited states are really a trial to both the exchange potential and the correlation potential because of possible high nonsymmetric atomic structures. Even for sophisticated ab initio methods, it is an ordeal to accurately calculate the electronic structures of triply excited states. In this section, we present total energies and Auger electron energies from our calculation for triply excited resonance states of Li-like ions. For these resonance states and Auger processes, there are neither HF results nor DFT results to compare to. We will compare our results to other theoretical results and experimental data. To our knowledge, this is the first time that the triply excited resonance states of Li-like ions have been calculated using the DFT method.

In Table XI, we present the total energies obtained from the XC calculation for triply excited resonance states of Lilike ions $(Z=3-10)$. Also given in this table are the theoretical results of the truncated diagonalization method (TDM) [43] and the multichannel saddle-point complex-rotation (MSPCR) method [51,52]. The numbers in the parentheses denote the absolute percentage deviations of our results with respect to the data followed by these numbers. It is shown that our results are in very good agreement with those of MSPCR. The absolute deviations are less than $0.95 \%$. Apart from $2 s 2 p^{2} P$ and $2 p^{32} P$ states, the discrepancies of the results of the TDM are less than $0.91 \%$. For $2 s 2 p^{2}{ }^{2} P$ and $2 p^{32} P$ states, the deviations are a little bit larger, but no more than $1.38 \%$. This demonstrates that our calculations for triply excited states are quite accurate. We noticed that for $2 s 2 p^{2} P$ and $2 p^{32} P$ states, our results are more negative than other theoretical results, which may be caused by the overestimations of correlation energy to these states.

One of the most important processes from the highly triply excited resonance states of Li-like ions is Auger electron emission via autoionization, leaving states of He-like ions [53,54]. The calculation of the Auger electron spectroscopy involves the total energies of the triply excited resonance states of Li-like ions given in Table XI and the total energies of the singly excited states of He-like ions listed in Table V. In Table XII, we report the calculated Auger electron energies for the triply excited resonances of Li-like ions. To examine the calculation precision for triply excited states, we also list, in Table XII, the theoretical results of the TDM
[43], MSPCR [51,52], and hyperspherical close-coupling (HSCC) methods [54], as well as the experimental results $[53,54]$. Again, the numbers in the parentheses denote the absolute percentage deviations of our results with respect to the data followed by the numbers. It is shown that apart from the processes related to the $1 s 2 p{ }^{1} P$ state in neutral $\mathrm{Li}$ the discrepancies of our results with respect to both the experimental results and other theoretical results are less than $0.95 \%$. Even for the processes involved in the $1 s 2 p{ }^{1} P$ state in neutral $\mathrm{Li}$, the deviations are no more than $1.5 \%$. This illustrates that our calculation of the triply excited states is quite accurate and the proposed approach with the SLHF exchange potential is efficient and effective to the multiply excited-state calculation.

In summary, based on the spin-dependent localized Hartree-Fock density functional method and Slater's diagonal sum rule, we present an approach for the calculation of highly and multiply excited states of atomic systems. In this approach, the KS exchange potential is replaced by an exact nonvariational SLHF exchange potential. The SLHF exchange potential qualifies for the calculation of the excited state because it provides a potential with free self-interaction, the correct long-range behavior, and the symmetry dependence of an atomic state. The procedure has been applied to the calculation of singly, doubly, and triply excited Rydberg states of He- and Li-like ions. The generalized pseudospectral method with nonuniform grids is used to discretize the spatial coordinates and to optimize the solutions of the KS equation to obtain accurate spin orbitals and orbital energies for both the ground state and excited states. X-only results are in very good agreement with HF results and X-only WF results, demonstrating that the proposed procedure is very accurate for the calculations of highly and multiply atomic excited states. The correlation effects are considered by incorporating the LYP correlation potential and the energy functional into calculation. The total energies from the XC calculation are close to the available sophisticated ab initio theoretical results and experimental data. The maximum discrepancy of our calculated energies to the available experimental results is less than $1.0 \%$, illustrating that the SLHF exchange potential combined with the LYP correlation potential is satisfactory for the excited-state calculation of atomic systems. Thus the procedure presented in this paper provides a simple and computationally efficient scheme for the accurate calculation of highly and multiply excited Rydberg states within the DFT.

\section{ACKNOWLEDGMENTS}

This work was partially supported by the Chemical Sciences, Geosciences and Biosciences Division of the Office of Basic Energy Sciences, Office of Science, U. S. Department of Energy, and by the National Science Foundation. We acknowledge the Kansas Center for Advanced Scientific Computing for the support of supercomputer time. 
[1] P. Hohenberg and W. Kohn, Phys. Rev. 136, B864 (1964).

[2] W. Kohn and L. J. Sham, Phys. Rev. 140, A1133 (1965).

[3] R. G. Parr and W. Yang, Density-Functional Theory of Atoms and Molecules (Oxford University Press, New York, 1989).

[4] R. M. Dreizler and E. K. U. Gross, Density Functional Theory: An Approach to the Quantum Many-body Problem (SpringerVerlag, New York, 1990).

[5] F. D. Sala and A. Görling, Phys. Rev. Lett. 89, 033003 (2002).

[6] A. D. Becke, Phys. Rev. A 38, 3098 (1988).

[7] C. Lee, W. Yang, and R. G. Parr, Phys. Rev. B 37, 785 (1988).

[8] J. P. Perdew and Y. Wang, Phys. Rev. B 33, 8800 (1986).

[9] J. P. Perdew and A. Zunger, Phys. Rev. B 23, 5048 (1981).

[10] X. M. Tong and S. I. Chu, Phys. Rev. A 55, 3406 (1997).

[11] M. Petersilka, U. J. Gossmann, and E. K. U. Gross, Phys. Rev. Lett. 76, 1212 (1996).

[12] M. E. Casida et al., J. Chem. Phys. 108, 4439 (1998).

[13] T. Grabo, M. Petersilka, and E. K. U. Gross, J. Mol. Struct.: THEOCHEM 501, 353 (2000).

[14] F. D. Sala and A. Görling, J. Chem. Phys. 115, 5718 (2001).

[15] R. Singh and B. M. Deb, Phys. Rep. 311, 47 (1999).

[16] M. K. Harbola and V. Sahni, Phys. Rev. Lett. 62, 489 (1989).

[17] V. Sahni, Y. Li, and M. K. Harbola, Phys. Rev. A 45, 1434 (1992).

[18] A. K. Roy and S. I. Chu, Phys. Rev. A 65, 052508 (2002).

[19] R. Singh and B. M. Deb, J. Chem. Phys. 104, 5892 (1996).

[20] F. D. Sala and A. Görling, J. Chem. Phys. 118, 10439 (2003).

[21] F. D. Sala and A. Görling, Int. J. Quantum Chem. 91, 131 (2003).

[22] T. Hupp, B. Engels, and A. Görling, J. Chem. Phys. 119, 11591 (2003).

[23] T. Hupp et al., Z. Phys. Chem. (Munich) 217, 133 (2003).

[24] O. V. Gritsenko and E. J. Baerends, Phys. Rev. A 64, 042506 (2001).

[25] J. C. Slater, Quantum Theory of Atomic Structure, Vol. II (McGraw-Hill, New York, 1960).

[26] J. Wang, S. I. Chu, and C. Laughlin, Phys. Rev. A 50, 3208 (1994).

[27] J. B. Krieger, Y. Li, and G. J. Iafrate, Phys. Rev. A 46, 5453 (1992).
[28] Y. Li, J. B. Krieger, and G. J. Iafrate, Phys. Rev. A 47, 165 (1993).

[29] J. D. Talman and W. F. Shadwick, Phys. Rev. A 14, 36 (1976).

[30] R. D. Cowan, The Theory of Atomic Structure and Spectra (University of California Press, Berkeley, 1981).

[31] X. M. Tong and S. I. Chu, Phys. Rev. A 64, 013417 (2001).

[32] X. Chu and S. I. Chu, Phys. Rev. A 63, 023411 (2001).

[33] X. M. Tong and S. I. Chu, Phys. Rev. A 61, 031401(R) (2000).

[34] C. Pollak, A. Rosa, and E. J. Baerends, J. Am. Chem. Soc. 119, 7324 (1997).

[35] C. F. Fischer, The Hartree-Fock Method for Atoms (Wiley, New York, 1977).

[36] A. D. Becke, J. Chem. Phys. 96, 2155 (1992).

[37] C. E. Moore, Ionization Potentials and Ionization Limits Derived from the Analysis of Optical Spectra, Natl. Bur. Stand. Ref. Data Ser. (U.S. GPO, Washington, D.C., 1970).

[38] A. A. Radzig and B. M. Smirnov, Reference Data on Atoms and Molecules (Springer, Berlin, 1985).

[39] A. Veillard and E. Clementi, J. Chem. Phys. 49, 2415 (1968).

[40] M. T. Carroll, R. F. W. Bader, and S. H. Vosko, J. Phys. B 20, 3599 (1987).

[41] A. Bürgers, D. Wintgen, and J. Rost, J. Phys. B 28, 3163 (1995).

[42] Y. K. Ho, J. Phys. B 12, 387 (1979).

[43] M. J. Conneely and L. Lipsky, At. Data Nucl. Data Tables 82, 115 (2002)

[44] W. A. Goddard III, Phys. Rev. 176, 106 (1968).

[45] J. S. Sims and S. A. Hagstrom, Phys. Rev. A 11, 418 (1975).

[46] G. Brual and S. M. Rothstein, J. Chem. Phys. 69, 1177 (1978).

[47] Y. K. Ho, Phys. Rev. A 48, 3598 (1993).

[48] L. Wu and J. Xi, J. Phys. B 23, 727 (1990).

[49] R. S. Oberoi, J. Phys. B 5, 1120 (1972).

[50] R. P. Madden and K. Codling, Astrophys. J. 141, 364 (1965).

[51] B. Gou and K. T. Chung, J. Phys. B 29, 6103 (1996).

[52] K. T. Chung and B.-C. Gou, Phys. Rev. A 52, 3669 (1995).

[53] M. Rødbro, R. Bruch and P. Bisgaard, J. Phys. B 12, 2413 (1979).

[54] M. Zamkov et al., Phys. Rev. A 67, 050703(R) (2003). 